\title{
Room Temperature Polyaniline-Polyvinylalcohol Composite-based flexible Ammonia Sensor
}

\author{
S. P. Patil ${ }^{1}$, S. A. Pethe ${ }^{2}$, J. B. Bhadane ${ }^{3}$, Y. V. Gulwadi ${ }^{4}$

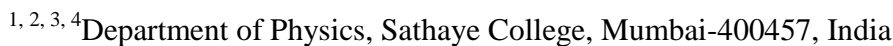

\begin{abstract}
In the present work, room temperature polyaniline-polyvinylalcohol (PANI-PVA) composite-based flexible ammonia sensor has been fabricated. The PANI-PVA composite films were synthesized by an in situ chemical polymerization of aniline in presence of PVA. The PANI-PVA composite-based flexible ammonia sensor shows better ammonia sensing properties than pure PANI. The sensor with 4\% PVA exhibits excellent sensing characteristics such as rapid response ( 50 s to 4000 ppm), fast recovery ( 90 s for 4000 ppm) and good reproducibility.
\end{abstract}

Keywords: Conducting Polymers, Gas Sensors, Polymer composites, Ammonia sensor, Flexible sensor

\section{Introduction}

Conducting polymer based gas sensors offer a promising alternative to semiconducting or other classical gas sensors because of the several advantages they offer such as- (a) remarkable sensitivity at room temperature, (b) low energy consumption and simplicity of device fabrication (c) facile property adjustment (d) low detection limits and (e) short response time. However, the disadvantages that hinder the wide commercial use of conducting polymer based sensors are: (a) relatively lower stability (b) low selectivity and (c) irreversibility of the gas response. Therefore considerable work needs to be carried out so as to understand several aspects related to the use of conducting polymers as sensing elements in the fabrication of gas sensors. There have been several reports on improving selectivity and stability of conducting polymers by making use of new methods, such as their synthesis in nano-structured forms ${ }^{[1]}$, or by addition of metal catalysts ${ }^{[2]}$ and by combination with other polymer [3]

One of the first practical sensors to detect ammonia was based on conducting polypyrrole ${ }^{[4]}$. However, its drawback was low sensitivity and irreversibility of gas response. Detailed investigation of sensitivity to different gases has been carried out in J. Miasik et. al ${ }^{[5]}$ who have demonstrated good reversibility of the response to ammonia in the range $100-10,000$ ppm, but the concentration dependence of the response was non-linear. Also, this polypyrrole sensor has low sensitivity and very long (tens of minutes) response time.

The authors of ${ }^{[6]}$ have demonstrated an ammonia sensor based on PANI films doped with nickel containing anions. These sensors were shown to operate in a wide (1-1000 ppm) ammonia concentration range with short response time (60-120 s). But their sensitivity is very low, wherein only a three fold rise of resistance was observed with a ten thousand fold increase in gas concentration. Another drawback of these sensors was the incomplete reproducibility of their reaction to ammonia. used for the preparation of gas sensing elements. But bulk production of thin films is limited by this method due to the complexity of current density control and the process of selection of an electrolyte ${ }^{[7]}$. Also, electrochemically prepared film is often brittle and not processable. Hence, in our work we used a simple and cheap method of preparing composite films of polyanline (PANI) with polyvinyl alcohol (PVA). These free standing PANI-PVA composite films were synthesized through an in situ chemical polymerization of aniline in presence of PVA. In the recent past, many research groups have investigated gas sensors based on conducting polymer-metal oxide composites ${ }^{[8,9]}$ but there have been only few reports on the use of conducting polymer combined with an insulating polymer (i.e. polymer composites) used in gas sensing, particularly at room temperature. In this study, the effect of ammonia gas on the resistance of PANI-polyvinyl alcohol (PVA) composite films was examined for the fabrication of room temperature operative $\mathrm{NH}_{3}$ gas sensor.

\section{Experimental}

\subsection{Preparation of the composite films}

Analytical reagent (AR) grade chemicals were used in the present study. The monomer aniline was procured from S. D. Fine Chemicals, Mumbai and it was distilled twice before being used for synthesis. Hydrochloric acid ( $\mathrm{HCl})$ and the oxidant ammonium persulphate were procured from EMerck (India) and were used as received. PANI-PVA composite films were prepared as per the procedure illustrated in Fig.1.

\subsection{Fabrication of the sensor device}

The sensor device was in the form of a two point probe consisting of two small copper plates fixed onto a Teflon holder. The composite film was fixed in between the two copper electrodes to which 2 wire contacts were made. The two wires were connected to an ohmmeter. This arrangement is depicted schematically in Fig.2.

The method of direct electrochemical synthesis of conducting polymers on an electrode has generally been 


\section{International Journal of Science and Research (IJSR) \\ ISSN (Online): 2319-7064 \\ Index Copernicus Value (2013): 6.14 | Impact Factor (2014): 5.611}

The changes in electrical resistance were monitored as a function of time using digital multimeter (SM-5015, Scientific, India) controlled by a personal computer.

\section{Result and Discussion}

\subsection{Response Behavior of the PANI-PVA Sensor}

The sensor response of the PANI-PVA composite films was measured in terms of the change in their resistance to different concentration of $\mathrm{NH}_{3}$ gas in ppm. Fig. 3 shows the responses for 4000, 8000 and 20,000 ppm of $\mathrm{NH}_{3}$ respectively. The response time is defined as the time required for the resistance to increase to $90 \%$ of the maximum value. The response time $t_{k}=50 \mathrm{~s}, 75 \mathrm{~s}$ and $30 \mathrm{~s}$ for 4000, 8000 and 20,000 ppm of $\mathrm{NH}_{3}$ gas respectively. These values indicate that the sensor responds rapidly to $\mathrm{NH}_{3}$ gas. Also, the sample recovers its initial value of resistance in 90s for $4000 \mathrm{ppm}, 7 \mathrm{~min}$. $20 \mathrm{~s}$ for $8000 \mathrm{ppm}$ whereas for $20,000 \mathrm{ppm}$ the recovery time is very long (about $1 \mathrm{~h}$ ). Thus, the sensor response is reversible only up to an optimum concentration of $\mathrm{NH}_{3}$ gas (16000 ppm) beyond which the recovery time is quite long.

The reversible nature of the sensor response up to a certain concentration of $\mathrm{NH}_{3}$ gas (in ppm) may be due to the weak nature of the chemical bonds between the ammonia molecules and the $\mathrm{N}^{+} \mathrm{H}^{-}$adsorption centres in the PANI film. Also, for a small amount of $\mathrm{NH}_{3}$ molecule, there is always a sufficient number of $\mathrm{N}^{+}-\mathrm{H}$ active adsorption centres in the PANI- PVA film, so that ammonia interaction with them will produce the rated response value. At very high concentration of $\mathrm{NH}_{3}$ gas ( $\geq 20,000 \mathrm{ppm}$ ), the resistance reaches a plateau value and this irreversibility of sensor response is due to the accumulation of ammonium residues in the sensitive layer. The slow desorption of ammonia from PANI-PVA layers seems to be attributable to the additional binding of $\mathrm{NH}_{4}^{+}$ions to specific PANI centres. Such a binding prevents ammonia molecules from interacting with a fraction of the PANI $\mathrm{N}^{+}-\mathrm{H}$ adsorption centres.

\subsection{Sensitivity to $\mathrm{NH}_{3}$ Gas:}

The sensitivity (or gas response) has been defined as

$$
S=\frac{R_{m}-R_{a}}{R_{a}} \times 100 \%
$$

where $\mathrm{R}_{\mathrm{m}}=$ Maximum resistance attained by film in presence of $\mathrm{NH}_{3}$ gas, $\mathrm{R}_{a}=$ Resistance of the film in air. Fig.3 shows the dependence of the sensitivity (S) on the $\mathrm{NH}_{3}$ concentration for the PANI-PVA composite films. The values of $S$ are found to be $24.48 \%$ for $4000 \mathrm{ppm}, 44 \%$ for $8000 \mathrm{ppm}$ and $67 \%$ for $20,000 \mathrm{ppm}$. The rapid response time and good sensitivity of the composite films can be attributed to the morphology and porosity of the films. ${ }^{[10]}$.

The morphology of the composite films consists of nanorods as revealed by SEM [Fig. 4]. The higher surface area of the film and smaller diameters of the rods gives a shorter response time because diffusion is faster.

\section{Conclusions}

The free standing polyaniline-polyvinyl alcohol (PANIPVA) composite films have been investigated for room temperature operative ammonia gas sensor. These films show better ammonia sensing properties than pure PANI. The PANI-PVA film with 4\% PVA exhibit excellent sensing characteristics such as rapid response, fast recovery and good reproducibility. This work demonstrates the potential of using PANI-PVA composite films as sensing material in the fabrication of $\mathrm{NH}_{3}$ sensors.

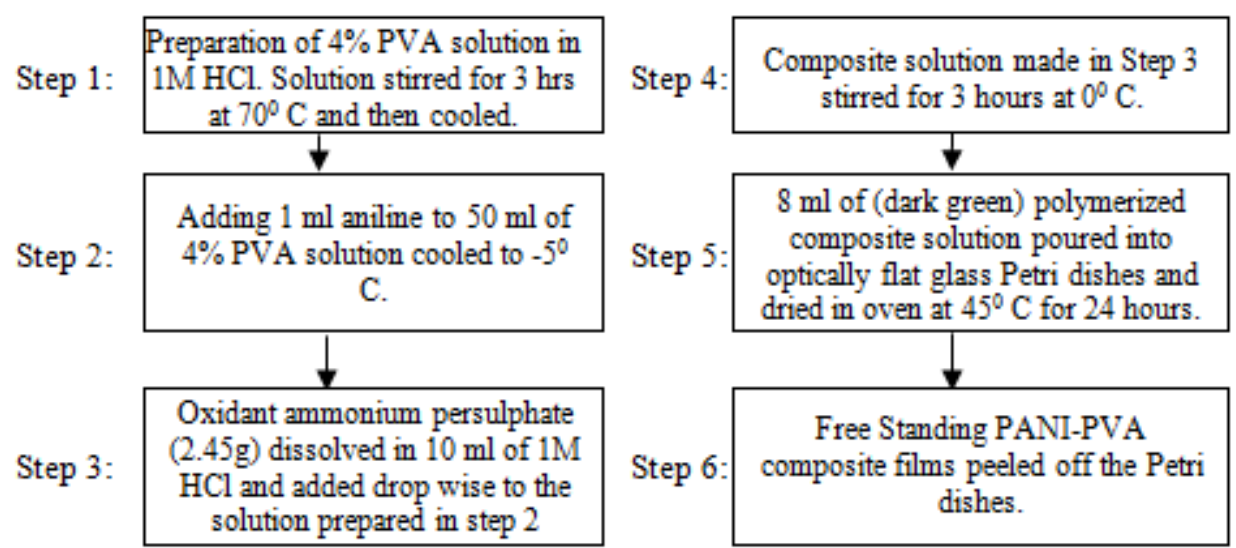

Figure 1: Preparation of PANI-PVA composite films

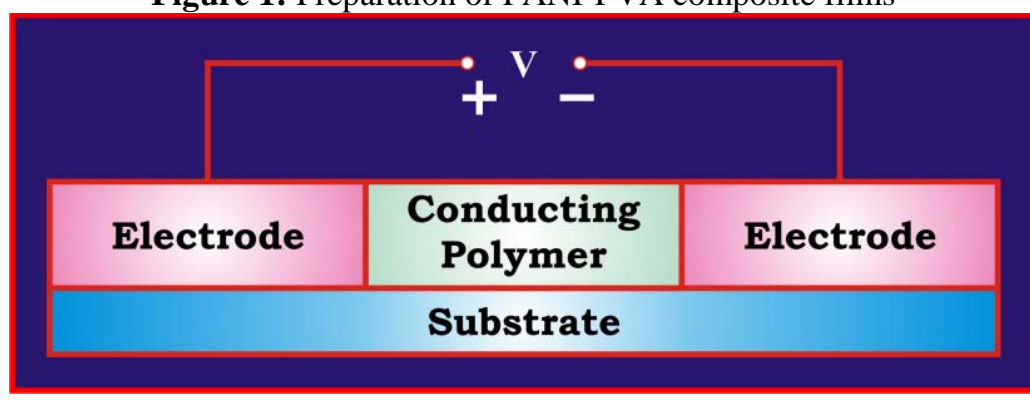

Figure 2: Schematic of the sensing device

Volume 5 Issue 3, March 2016 


\section{International Journal of Science and Research (IJSR)
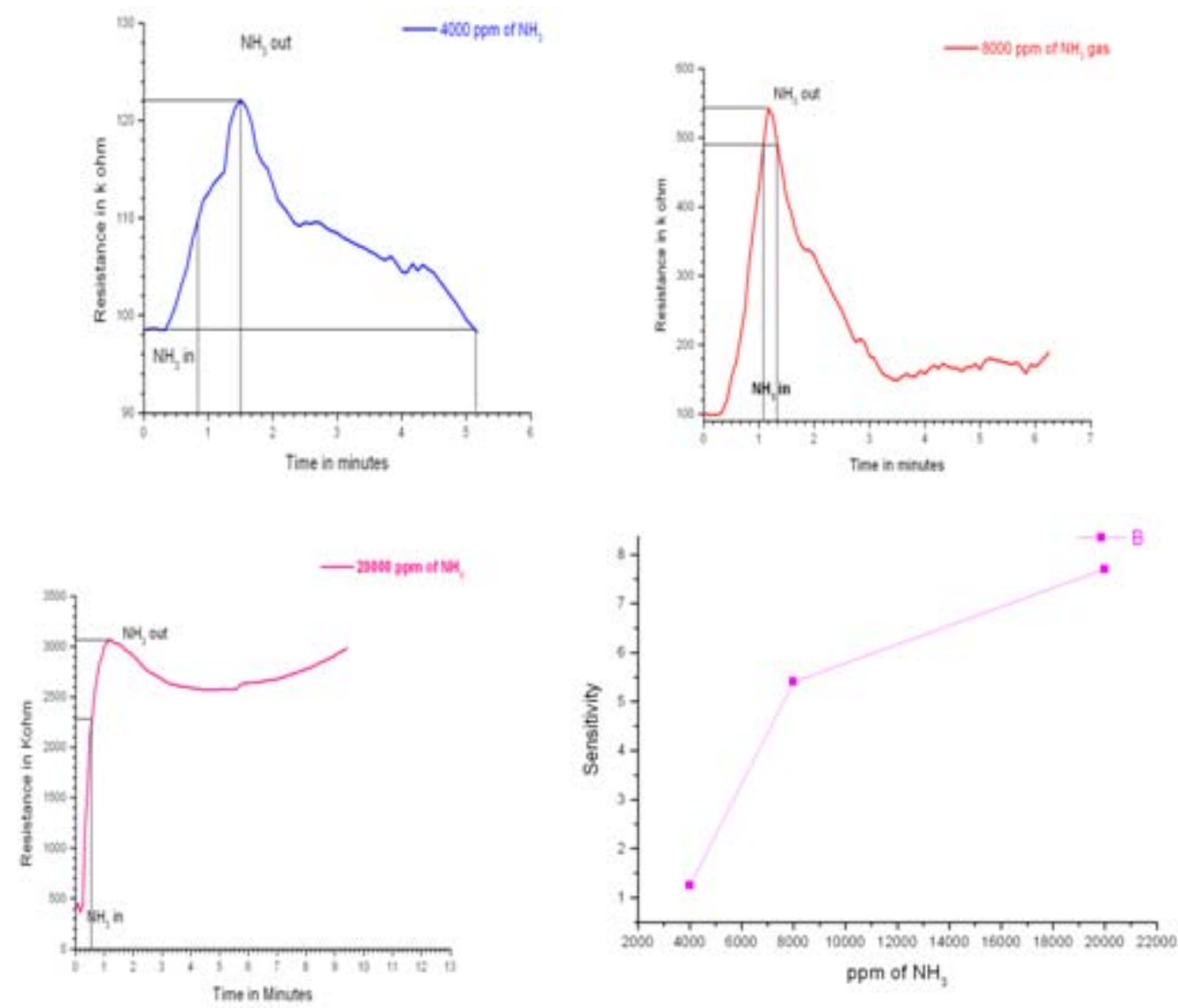

Figure 3: Responses of PANI-PVA based sensor to 4000, 8000 and 20000 ppm of $\mathrm{NH}_{3}$ gas and dependence of the sensitivity (S) on the $\mathrm{NH}_{3}$ concentration.

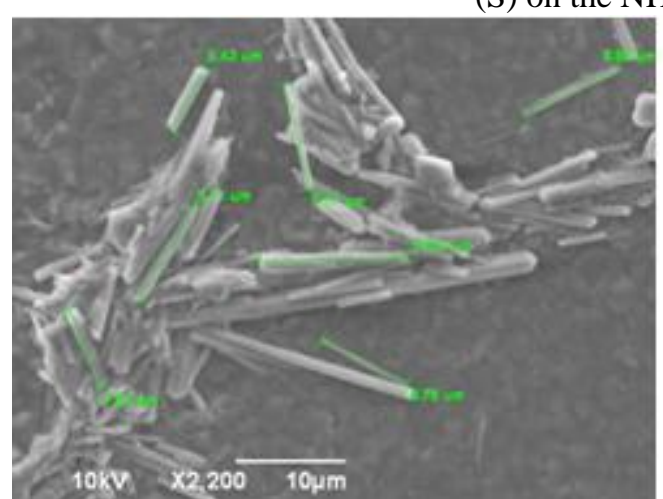

Figure 4: SEM images of PANI-PVA composite films

\section{References}

[1] A. Macagnano, E. Zampetti, S.Pantalei, F.De. Cesare, A. Bearzotti, K.C. Persaud, Thin Solid Films 520 (2011) 978

[2] Z. F. Li, F. D. Blum, M. F. Bertino, C. S. Kim, Sens. Actuators B, 161,(2012), 390

[3] T. Tonosaki, T.Oho, K. Isomura, K. Ogura, J. Electroanal. Chem,20(2002), 89

[4] C. Nylander, $M$ Armgarth and I. Lundstrom, An ammonia detector based on a conducting polymer, Anal. Chem. Symp. Ser, 17 (1983), 203-207

[5] J. Miasik, A. Hopper and B. Tofield, Conducting polymer gas sensors, J.Chem. Soc, Farrady Trans. I 82b(1986), 1117

[6] S. A. Krutovertsev, S. I. Sorokin, A.V. Zorin, Y. A. Letuchy and O. Yu. Antonova, Polymer film based

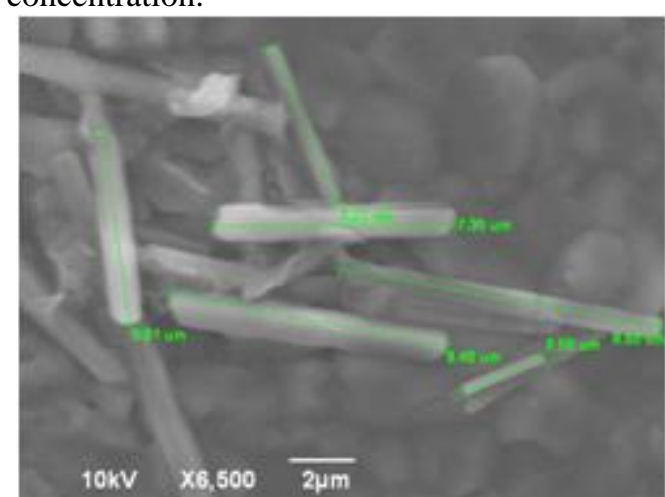

sensors for ammonia detection, Sens. Actuators, B. 7 (1992) 492-494

[7] M. Matsuguchi, J. Io, G. Sugiyama, Y. Sakai Synth. Metals, 128 (2002) 15-19

[8] S Kanefusa and N Haradome,Unique phenomenon in $\mathrm{SbO}_{2}$ gas based gas sensing devices exposed to ammonia gas, Solid state Electron, 27(1984) 533.

[9] Z.F.Li, F.D Blum, M.F. Bertino, C.S. Kin, Sens. Actuators B, 161 (2012) 390.

[10] Polyaniline nanofiber gas sensors; S. Virji, J. Huang, R. B. Kaner, B. H. Weiller. Nanoletters (2004, Vol-4, No-3, 491-496 